\title{
Lipidated $\beta$-lactamases: from bench to bedside
}
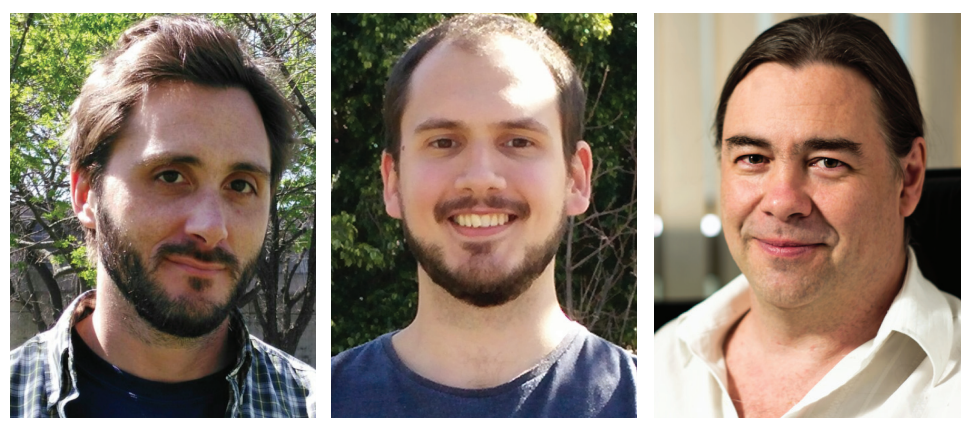

Lisandro J González ${ }^{\ddagger 1,2}$, Guillermo Bahr ${ }^{\ddagger, 1,2}$ \& Alejandro J Vila ${ }^{*, 1,2}$

First draft submitted: 31 August 2016; Accepted for publication: 5 October 2016;

Published online: 10 November 2016

Carbapenemases represent one of the largest clinical threats to the action of carbapenems; the last resort drugs for the treatment of healthcare-associated infections caused by Gram-negative bacteria [1]. Metallo- $\beta$-lactamases (MBLs) are $\mathrm{Zn}$ (II)-dependent enzymes that constitute the largest family of carbapenemases of clinical impact. Among them, the New Delhi Metallo- $\beta$-lactamase (NDM- 1 ) is a plasmid-encoded carbapenemase that has experienced the fastest and widest geographical spread, having been detected in more than 80 countries worldwide since its identification in 2008 [2]. Remarkably, the clinical success of this resistance determinant does not seem to be associated with the dissemination of a particular clone or genetic structure [3]. We have recently suggested that this particular success is due to the cellular localization of NDM-1: while all other known MBLs are soluble periplasmic proteins, NDM-1 is a lipoprotein anchored to the inner leaflet of the outer membrane in Gram-negative bacteria [4]. Despite NDM-1 being reported as a lipidated protein in 2011 [5], this fact was regarded as a biochemical curiosity and deserved little attention until recently when we reported that lipidation and membrane anchoring confer unique evolutionary advantages to NDM-1 [4].

\section{Protein lipidation in bacteria}

The mechanism of bacterial protein lipidation is a well-characterized process and has been extensively reviewed elsewhere [6]. Protein lipidation takes place by covalent attachment of acyl moieties to a cysteine residue at the $\mathrm{N}$-terminus, that enable anchoring of globular, hydrophilic proteins to the phospholipids of the bacterial

\section{KEYWORDS}

- antibiotic resistance

- $\beta$-lactamases $\bullet$ lipoproteins

- membrane-anchoring

- NDM-1 


\author{
"The bro-1 gene is \\ characterized by a \\ significantly lower $\mathrm{G}+\mathrm{C}$ \\ content (31\%) than the \\ Moraxella catarrhalis \\ genome (42\%)."
}

membrane. This allows lipoproteins to be membrane-anchored but at the same time preserving their functionality in an aqueous environment. This post-translational modification, first identified in the Escherichia coli protein Lpp (also known as Braun's lipoprotein), is present in hundreds of bacterial proteins with diverse roles encompassing enzymatic activity, transport, virulence and structural integrity of the bacterial envelope [6]. Indeed, approximately $1-3 \%$ of the bacterial genome codes for lipoproteins, many of them with unknown function.

Bacterial protein lipidation depends on a processing system located in the cytoplasmic membrane, widely conserved in both Gram-negative and Gram-positive organisms [6]. Prelipoprotein precursors synthesized in the cytosol are generally exported by the secretion ( $\mathrm{Sec}$ ) pathway, though there are instances of export through the twin-arginine translocation (Tat) system. After translocation, proteins containing a lipidation signal (lipobox) are first modified by the addition of a diacylglycerol moiety to the sulfhydryl group of the conserved cysteine residue within the lipobox, catalyzed by the Lgt enzyme. This diacylated protein is then processed by the lipoprotein signal peptidase LspA, which cleaves the protein within the lipobox at the peptide bond immediately preceding the lipidated cysteine residue. The free amine moiety of the now $\mathrm{N}$-terminal cysteine is acted upon by the transacylase Lnt, which binds an acyl group to it by an amide linkage.

In Gram-negative bacteria, lipoproteins are by default translocated to the outer membrane by the LolABCDE system [6]. LolCDE is an ATP-binding cassette which extracts lipidated proteins from the inner membrane and transfers them to LolA, a periplasmic protein which shuttles its acylated substrates to the outer membrane. Finally, LolB, itself an outer membrane lipoprotein, receives this cargo from LolA and deposits it into the inner leaflet of the outer membrane. Lipoproteins that remain in the inner membrane possess a signal to avoid the LolCDE system, generally located at positions +2 to +4 from the lipidated cysteine.

\section{Lipidated lactamases}

Lactamases bound to the cell surface were first reported in Gram-positive organisms more than 50 years ago [7], and were later demonstrated to be membrane-anchored proteins containing lipobox sequences within their leader peptides [8]. These lipidated enzymes are class A serine- $\beta$-lactamases: BLIII (also known as BlaZ) from Bacillus cereus [9], PC1 from Staphylococcus aureus [8] and PenP from Bacillus licheniformis [10], with numerous homologs in related organisms readily found in the GenBank database. In Gram-positive bacteria, lacking a periplasmic space, membrane anchoring would prevent the enzyme from being released into the growth medium, thus keeping the lactamase associated to the cell. Despite the advantage of this anchoring, up to $50 \%$ of the total activity of BLIII and PenP lactamases can be detected in culture supernatants $[9,10]$, and a variable percentage of activity has been also observed for PC1 and its homologs in the soluble form [11]. These 'exoenzyme' forms are generated by proteolytic cleavage of the lipidated enzyme, resulting in a soluble lactamase of lower molecular weight than the membrane-anchored counterpart [8].

Lipidated lactamases are less common among Gram-negative bacteria, as may be expected from the presence of an outer membrane which maintains soluble enzymes associated to the cell. The most characterized is the serine $\beta$-lactamase BRO-1, from the opportunistic respiratory pathogen Moraxella catarrhalis [12]. Despite displaying a canonical lipobox, only $10 \%$ of BRO-1 is membrane-bound in its natural host. Instead, when expressed in E. coli, $45 \%$ of the enzyme is attached to the membrane [13]. In contrast, NDM-1 [4] and Burkholderia pseudomallei PenA [14] were recently described to be completely localized in membrane fractions.

The bro- 1 gene is characterized by a significantly lower $\mathrm{G}+\mathrm{C}$ content $(31 \%)$ than the M. catarrhalis genome (42\%). Bootsma et al. suggested that $\beta$-lactamase lipoproteins derive from Gram-positive organisms, since all known enzymes at the moment belonged to that group of bacteria [12]. This is not the case for pen $A$ and $b l a_{\mathrm{NDM}-1}$, with much higher $\mathrm{G}+\mathrm{C}$ contents (70 and $62 \%$, respectively). In the case of $\operatorname{pen} A$, the $\mathrm{G}+\mathrm{C}$ content matches that of the bacterial host genome.

While the membrane anchoring of $\beta$-lactamases in Gram-positive bacteria seems to provide an advantage in terms of enzyme localization and association of the lactamase activity to the cell, the role of lipidation from enzymes in Gram-negative organisms is less clear. Our recent work demonstrated that the membrane anchoring of NDM-1, which is a 
zinc-dependent enzyme, helped prevent its degradation in the periplasmic space upon metal starvation in the extracellular milieu, and occurs with a soluble mutant of the enzyme [4]. This gain in stability is of great importance for maintaining carbapenem resistance during pathogenesis, since the immune system secretes large amounts of a $\mathrm{Zn}$ (II)-chelator protein, calprotectin, in the sites of infection. In this way, lipid modification confers additional fitness to this MBL by augmenting its stability in vivo.

Membrane anchoring also favors secretion of the lactamases into outer membrane vesicles (OMVs) [4,15]. This has been shown to be the case for NDM-1 and possibly BRO-1. OMVs are released by both pathogenic and nonpathogenic Gram-negative bacteria in a ubiquitous process that occurs over the course of normal growth [16]. OMVs are formed by fission of outer membrane protrusions that enclose periplasmic components in a yet uncharacterized process. These membrane-enclosed 'bacterial packs' have been associated with cellular communication and bacterial pathogenesis. We showed that OMVs containing NDM-1 can protect nearby populations of bacteria from otherwise lethal antibiotic levels by vesicle-mediated transfer of the enzyme [4]. Inclusion into OMVs not only allows secretion of NDM-1, but also protects the enzyme from extracellular proteases or $\mathrm{Zn}$ (II) depletion as it resides in the lumen of the vesicles. We also showed that OMVs from clinical pathogens expressing NDM-1 can carry both the protein and the gene coding for it, supporting the notion that OMVs can expand the options for horizontal transfer. Overall, membrane anchoring is able to stabilize a protein scaffold and facilitate secretion of enzymes in the extracellular milieu.

Despite NDM-1 is the only characterized membrane-bound MBL, we have reported the existence of numerous endogenous MBL homologs containing a lipobox sequence sharing a high degree of homology with different groups of characterized soluble MBLs [4]. These putative lipidated MBLs derived mostly from functional metagenomics analysis of soil bacteria [17], suggesting that the environmental microbiota constitute a reservoir of membraneanchored MBLs. As in the case of NDM-1, these lipidated MBLs may have an enhanced potential for dissemination, helped by the transfer of $\beta$-lactamase protein via OMVs, which protects antibiotic-sensitive bacteria.

\section{Bacterial protein lipidation as a target}

Bacterial protein lipidation remains an unexploited antibiotic target for clinical treatment of infections. Globomycin, a cyclic lipopeptide from Streptomyces sp., inhibits lipoprotein signal peptidase LspA, leading to accumulation of unprocessed prolipoproteins in the inner membrane of E. coli, followed by cell death [18]. Both globomycin and a functional analog compound from Myxococcus xanthus were proposed as promising leads for the development of a new class of antibiotics [19]. Apart from the LspA peptidase, compounds that target the Lol lipoprotein translocation system have also been identified (recently reviewed by Brown [20]). In both cases, the blocking of the lipidation pathway prevents lipoproteins from reaching the outer membrane, which is lethal to the cell. As a result, these drugs not only offer new effective antibiotic targets not present in eukaryotic organisms, but also provide a mechanism to retain membrane-anchored lactamases in the inner membrane, avoiding their secretion in OMVs.

Knowledge of $\beta$-lactamase lipidation has a direct impact in the detection of carbapenemases in clinical pathogens. Membrane anchoring thwarts NDM-1 detection using the modified Hodge test, by preventing the release of the enzyme into the extracellular medium where carbapenemase activity is detected [21]. This problem can be sorted out by addition of a detergent to the Petri dish, which dissolves the enzyme and enables NDM-1 detection without false positives [21]. This provides an excellent example of how basic knowledge of molecular features can be translated directly to a clinical test. We anticipate that lipidated $\beta$-lactamases may become increasingly prevalent, and efforts in developing new tests and drugs will be required.

\section{Financial \& competing interests disclosure}

$G$ Bahr is recipient of a doctoral fellowship from Consejo Nacional de Investigaciones Cientificas y Técnicas (CONICET). LJ González and AJ Vila are staff members from CONICET. This work was supported by grants from Agencia Nacional de Promoción Cientifica y Tecnológica and the US NIH (1R01AI100560) to AJ Vila. The authors have no other relevant affiliations or financial involvement with any organization or entity with a financial interest in or financial conflict with the subject matter or materials discussed in the manuscript apart from those disclosed.

No writing assistance was utilized in the production of this manuscript. 


\section{References}

1 Patel G, Bonomo RA. 'Stormy waters ahead': global emergence of carbapenemases. Front.

Microbiol. 4, 48 (2013).

2 Kumarasamy KK, Toleman MA, Walsh TR et al. Emergence of a new antibiotic resistance mechanism in India, Pakistan, and the UK: a molecular, biological, and epidemiological study. Lancet Infect. Dis. 10, 597-602 (2010).

3 Poirel L, Dortet L, Bernabeu S, Nordmann P. Genetic features of blaNDM-1-positive Enterobacteriaceae. Antimicrob. Agents Chemother. 55, 5403-5407 (2011).

4 González LJ, Bahr G, Nakashige TG et al. Membrane anchoring stabilizes and favors secretion of New Delhi metallo- $\beta$-lactamase. Nat. Chem. Biol. 12, 516-522 (2016).

5 King D, Strynadka N. Crystal structure of New Delhi metallo- $\beta$-lactamase reveals molecular basis for antibiotic resistance. Protein Sci. 20, 1484-1491 (2011).

6 Kovacs-Simon A, Titball RW, Michell SL. Lipoproteins of bacterial pathogens. Infect. Immun. 79, 548-561 (2011).

7 Pollock MR. The cell-bound penicillinase of Bacillus cereus. J. Gen. Microbiol. 15, 154-169 (1956).

8 Nielsen JB, Lampen JO. Membrane-bound penicillinases in Gram-positive bacteria. J. Biol. Chem. 257, 4490-4495 (1982).
9 Nielsen JB, Lampen JO. $\beta$-lactamase III of Bacillus cereus 569: membrane lipoprotein and secreted protein. Biochemistry 22, 4652-4656 (1983).

10 Nielsen JB, Caulfield MP, Lampen JO. Lipoprotein nature of Bacillus licheniformis membrane penicillinase. Proc. Natl Acad. Sci. USA 78, 3511-3515 (1981).

11 East AK, Dyke KG. Cloning and sequence determination of six Staphylococcus aureus $\beta$-lactamases and their expression in Escherichia coli and Staphylococcus aureus. J. Gen. Microbiol. 135, 1001-1015 (1989).

12 Bootsma HJ, Aerts PC, Posthuma G et al. Moraxella (Branhamella) catarrhalis $\mathrm{BRO}$ $\beta$-lactamase: a lipoprotein of gram-positive origin? J. Bacteriol. 181, 5090-5093 (1999).

13 Bootsma HJ, van Dijk H, Verhoef J, Fleer A, Mooi FR. Molecular characterization of the BRO $\beta$-lactamase of Moraxella (Branhamella) catarrhalis. Antimicrob. Agents Chemother. 40, 966-972 (1996).

14 Randall LB, Dobos K, Papp-Wallace KM, Bonomo RA, Schweizer HP. MembraneBound PenA $\beta$-lactamase of Burkholderia pseudomallei. Antimicrob. Agents Chemother. 60, 1509-1514 (2016).

15 Schaar V, Nordström T, Morgelin M, Riesbeck K. Moraxella catarrhalis outer membrane vesicles carry $\beta$-lactamase and promote survival of Streptococcus pneumoniae and Haemophilus influenzae by inactivating amoxicillin. Antimicrob. Agents Chemother. 55, 3845-3853 (2011).

16 Schwechheimer C, Kuehn MJ. Outermembrane vesicles from Gram-negative bacteria: biogenesis and functions. Nat. Rev. Microbiol. 13, 605-619 (2015).

17 Forsberg KJ, Patel S, Gibson MK et al. Bacterial phylogeny structures soil resistomes across habitats. Nature 509 , 612-616 (2014).

18 Hussain M, Ichihara S, Mizushima S. Accumulation of glyceride-containing precursor of the outer membrane lipoprotein in the cytoplasmic membrane of Escherichia coli treated with globomycin. J. Biol. Chem. 255, 3707-3712 (1980).

19 Walsh CT, Wencewicz TA. Prospects for new antibiotics: a molecule-centered perspective. J. Antibiot. (Tokyo) 67, 7-22 (2014).

20 Brown DG. Drug discovery strategies to outer membrane targets in Gram-negative pathogens. Bioorg. Med. Chem. (2016) (In press).

21 Pasteran F, González LJ, Albornoz E et al Triton Hodge test: improved protocol for modified Hodge test for enhanced detection of NDM and other carbapenemase producers. J. Clin. Microbiol. 54, 640-649 (2016). 